\title{
Effects of diabetic keratopathy on corneal optical density, central corneal thickness, and corneal endothelial cell counts
}

\author{
FENG GAO, TAO LIN and YINGZHE PAN \\ Department of Ophthalmology, Xiangyang Hospital Affiliated to Hubei University of Medicine, \\ Xiangyang, Hubei 441000, P.R. China \\ Received January 18, 2016; Accepted July 5, 2016
}

DOI: $10.3892 /$ etm.2016.3511

\begin{abstract}
Diabetic keratopathy is an ocular complication that occurs with diabetes. In the present study, the effect of diabetic keratopathy on corneal optical density, central corneal thickness, and corneal endothelial cell count was investigated. One hundred and eighty diabetic patients (360 eyes) were enrolled in the study during the period from March, 2012 to March, 2013. The patients were divided into three age groups: $<5,5-10$ and $>10$ years, with 60 patients per group (120 eyes). During the same period, 60 healthy cases (120 eyes) were selected and labeled as the normal control group. The Pentacam was used to measure the corneal optical density, and central corneal thickness. Specular microscopy was used to examine the corneal endothelial cell density. The coefficient of partial correlation was used to control age and correlate the analysis between the corneal optical density, corneal endothelial cell density, and central corneal thickness. The stage of the disease, the medial and intimal corneal optical density and central corneal thickness was analyzed in the diabetes group. The corneal optical density in the diabetes group increased compared with that of the normal control group. The medial and intimal corneal optical density and central corneal thickness were positively correlated with the course of the disease. However, the corneal endothelial cell density was not associated with the course of diabetes. There was a positive association between the medial and intimal corneal optical density and central corneal thickness of the diabetic patients. In conclusion, the results of the present study show that medial and intimal corneal optical density and central corneal thickness were sensitive indicators for early diabetic keratopathy.
\end{abstract}

Correspondence to: Dr Yingzhe Pan, Department of Ophthalmology, Xiangyang Hospital Affiliated to Hubei University of Medicine, 15 Jiefang Road, Xiangyang, Hubei 441000, P.R. China

E-mail: panyz827@163.com

Key words: diabetes, diabetic keratopathy, corneal optical density, central corneal thickness, corneal endothelial cells, Pentacam

\section{Introduction}

Diabetic keratopathy is an ocular complication that occurs with diabetes. Previous findings showed that $47-64 \%$ of diabetics may be affected by primary keratopathy (1). Long-term hyperglycemia affects every structure of the patients' cornea, including recurrent corneal ulcer, persistent corneal epithelial defect, reduced sensitivity, corneal edema, corneal opacity and endothelial fluorescence leakage (2). However, there is a lack of evidence on diabetic keratopathy primarily because of a lack of effective diagnostic methods, particularly in the early period when the patients exhibit no classical symptoms and conventional slit-lamp did not identify abnormalities (2). It was also challenging to quantitatively detect and repetitively measure the abnormal changes of the cornea.

Corneal optical density is used to describe the biological and histological characteristic of the cornea. Corneal optical density, as unique biological and histological material, is closely associated with corneal transparency and may be used to describe the degree of corneal transparency (3). Previous findings showed that the corneal optical density in the area of inflammation was higher than that of the normal corneal optical density, even when the damages were repaired (one month later). Thus, corneal optical density is used to examine the inflammatory reaction and guide objective examination after corneal surgery (3). Pentacam is a camera that was designed on the basis of the Scheimpflug theory. Pentacam is capable of obtaining a three-dimensional image to evaluate various parameters, including the cornea, crystalline lens, and atria (4-6). It has been confirmed that Pentacam objectively assesses the nubecula through a quantitative measurement of cornea density (7).

In the present study, the Pentacam was used to detect the corneal optical density of the diabetic patients and alterations of transparency on diabetic mellitus patients during the disease were examined. A correlation analysis was subsequently conducted between the central corneal thickness and corneal endothelial cell density. The results offer a new course for early diagnosis and the pathogenesis of diabetic keratopathy.

\section{Materials and methods}

Materials. In total, 180 diabetic (360 eyes) patients, treated at the Department of Ophthalmology at the Xiangyang Hospital 


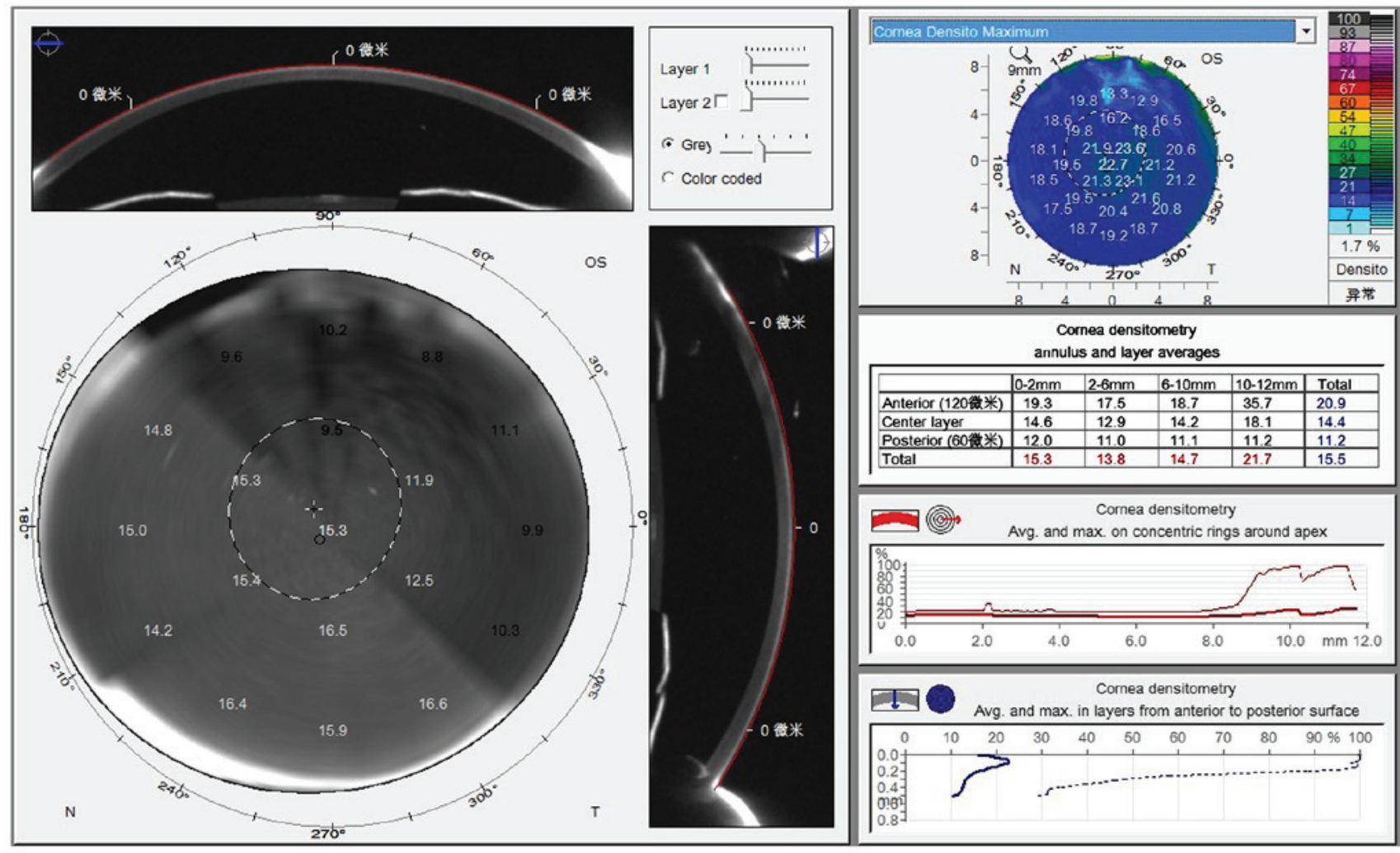

Figure 1. Corneal optical density under Pentacam detection.

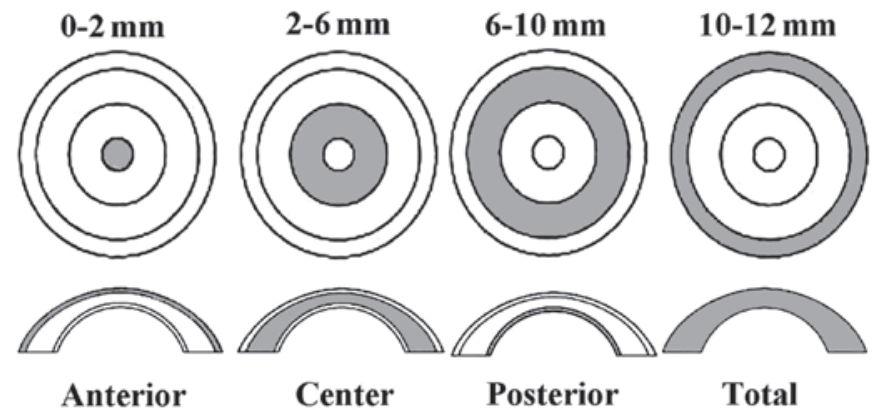

Figure 2. Region and layer of corneal optical density detection under Pentacam.

Affiliated to Hubei University of Medicine (Hubei, China) from March, 2012 to March, 2013 were enrolled in the present study. There were 94 male and 86 female patients, aged 41-77 years, with an average age of 59.27 years. The course of diabetes was between 1 and 20 years, with an average of 9.02 years. Simultaneously, another 60 healthy cases (120 eyes) were enrolled in the study as the normal control group. There were 26 male and 34 female subjects, aged $41-75$ years, with an average age of 59 and 17 years. The differences in age and gender between the two groups had no statistical significance $(\mathrm{P}>0.05)$. Patients with a history of eye surgery, laser treatment, contact lens wearing, eye traumas, keratonosus, uvea disease and intraocular hypertension disease were excluded from the study. All patients accepted to undergo a split-lamp examination and were confirmed as normal without any lesions in the eye. The diabetes group was divided into the $<5,5-10$ and $>10$ groups, each with 60 patients (120 eyes).
The study was approved by the ethics committee of Xiangyang Hospital Affiliated to Hubei University of Medicine. Written informed consent was obtained from the patients and/or guardians.

Corneal optical density and corneal thickness. The Pentacam (OCULUS Optikgeräte GmbH, Wetzlar, Germany) was used for the examination. Patients remained in a dark room for 5-10 min during the examination and accepted to be tested under the natural state of the pupil. The patients were kept in a dark room, asked to be seated and fix their lower jaw on a mandible support and stare at the fixation target in the center of the blue streak of the Pentacam. Patients were not allowed to blink or move their eyes. The examiner chose the measurement pattern ( 25 sections/sec) to scan automatically and obtained the data in $2 \mathrm{sec}$ in a non-contact manner. After the examination, the examiner manually measured the corneal density, and recorded the maximum density value on the vertical axis at the corneal vertex, using the gray value to represent the corneal density and propose 0 as complete transparency, and 100 as total nubecula. Simultaneously, the Pentacam was used to measure the minimum thickness of the central cornea (Figs. 1 and 2).

Corneal endothelial cell count. The EM-3000 fully-automatic corneal endothelial cell analyzer produced by TOMEY Corp. (Nagoya, Japan) was used to detect the amount of corneal endothelial cells. The patients were required to stare at the indicator light. The examiner adjusted the corneal endothelial meter to capture images of the corneal endothelium automatically. Its built-in computer analysis system was used to analyze 
Table I. Corneal optical density of the normal control group.

\begin{tabular}{|c|c|c|c|c|c|}
\hline Control & $0-2 \mathrm{~mm}$ & $2-6 \mathrm{~mm}$ & $6-10 \mathrm{~mm}$ & $10-12 \mathrm{~mm}$ & Total \\
\hline Anterior & $22.87 \pm 2.91$ & $21.32 \pm 2.80$ & $26.37 \pm 8.67$ & $35.69 \pm 11.22$ & $25.81 \pm 5.14$ \\
\hline Center & $14.63 \pm 1.62$ & $13.69 \pm 1.69$ & $19.68 \pm 6.78$ & $24.90 \pm 6.50$ & $17.47 \pm 3.60$ \\
\hline Posterior & $12.75 \pm 1.46$ & $12.03 \pm 1.55$ & $16.92 \pm 5.10$ & $21.75 \pm 5.74$ & $15.39 \pm 2.99$ \\
\hline Total & $14.77 \pm 1.46$ & $15.81 \pm 1.97$ & $20.81 \pm 6.74$ & $27.36 \pm 7.47$ & $19.74 \pm 3.89$ \\
\hline
\end{tabular}

Table II. Corneal optical density of the diabetics.

\begin{tabular}{llllll}
\hline DM & $0-2 \mathrm{~mm}$ & $2-6 \mathrm{~mm}$ & $6-10 \mathrm{~mm}$ & $10-12 \mathrm{~mm}$ & Total \\
\hline Anterior & $25.32 \pm 5.71$ & $21.48 \pm 3.73$ & $27.18 \pm 11.39$ & $35.79 \pm 13.71$ & $28.04 \pm 10.83$ \\
Center & $19.80 \pm 7.18$ & $16.30 \pm 6.19$ & $28.73 \pm 10.43$ & $35.03 \pm 13.42$ & $23.62 \pm 10.11$ \\
Posterior & $30.75 \pm 9.476$ & $27.79 \pm 12.35$ & $31.72 \pm 12.04$ & $37.37 \pm 11.06$ & $29.75 \pm 11.38$ \\
Total & $15.11 \pm 3.05$ & $18.01 \pm 6.53$ & $23.55 \pm 09.58$ & $28.72 \pm 10.91$ & $21.52 \pm 5.58$ \\
\hline
\end{tabular}

the endothelial images, calculate and print the results. The procedures were completed by the same professional examiner to reduce intraobserver bias. After controlling the age factors by partial correlation coefficient in the diabetes group, the relationship between the corneal optical density and course of diabetes, corneal optical density and number of endothelium, corneal optical density and corneal thickness were measured.

Statistical analysis. SPSS 18.0 software (Chicago, IL, USA) was used to analyze the data of the different groups. After the age factors were excluded, a covariance analysis was applied to test the series of data.P $<0.05$ was considered statistically significant.

\section{Results}

Corneal optical density value, central corneal thickness and corneal endothelial cell density of the diabetics and normal controls. In the two groups, the corneal optical density increased as the distance from the center increased. The corneal optical density of the same cornea gradually decreased from the epithelium of the outer cornea to the corneal endothelium. Compared to the normal control group, the corneal optical density and central corneal thickness of the diabetics increased, while the corneal endothelial cell density decreased $(\mathrm{P}<0.05)$ (Tables I and II).

Association between the course of diabetes and central corneal thickness, corneal endothelial cell count and corneal optical density. As the duration of diabetes was extended, the corneal optical density and central corneal thickness increased while the corneal endothelial cell density decreased. The result of the correlation analysis between the course of diabetes and the central corneal thickness, corneal endothelial cell number and corneal optical density showed that the central corneal thickness and the medial corneal optical density were positively correlated with the duration of diabetes $(\mathrm{P}<0.05)$.
Table III. Comparison of the central corneal thickness, corneal endothelial cell density, and corneal optical density (0-2 mm) on diabetics at different stages of the disease.

\begin{tabular}{lccc}
\hline Item & $<5$ years & $5-10$ years & $>10$ years \\
\hline CCT & $557.21 \pm 3.51$ & $569.41 \pm 4.01$ & $581.72 \pm 3.17$ \\
Cell density & $3015 \pm 34.92$ & $2401 \pm 26.74$ & $2092.58 \pm 52.36$ \\
Anterior & $25.28 \pm 4.94$ & $24.97 \pm 5.61$ & $25.41 \pm 4.98$ \\
Center & $16.20 \pm 2.56$ & $18.97 \pm 4.83$ & $22.36 \pm 6.05$ \\
Posterior & $20.37 \pm 7.61$ & $34.29 \pm 9.84$ & $41.02 \pm 11.36$ \\
Total & $14.75 \pm 3.49$ & $15.08 \pm 6.62$ & $16.03 \pm 5.37$ \\
\hline
\end{tabular}

CCT, central corneal thickness.

However, the corneal endothelial cell density, superficial and total corneal optical density were not significantly associated with the duration of diabetes $(\mathrm{P}>0.05)$ (Tables III-VI).

\section{Discussion}

Improvement in living standards and changes to lifestyle have led to an increase in the incidence of diabetes (6). Long-term hyperglycemia has toxic effects on all cells in the body, and its most profound effects on eye tissues are on the cornea and retina. Seventy percent of diabetes is complicated by keratopathy, including recurrent corneal epithelial erosions, delayed wound healing, ulcers and edema (1). Corneal nervous lesion diabetes, which would result in the loss of corneal sensitivity and innervation, may be associated with corneal epithelial erosion. Thus, corneal neuropathy is considered an important reason for corneal epithelial defects. Current studies on diabetic ocular complications are focused on diabetic retinopathy and diabetic cataract. To the best of our knowledge, few 
Table IV. Correlation between the stage of diabetes and central corneal thickness, corneal endothelial cell density, and corneal optical density (0-2 mm).

\begin{tabular}{lcccccr}
\hline Item & CCT & Cell density & Anterior & Center & Posterior & Total \\
\hline Course of disease & 0.570 & -0.032 & 0.420 & 0.295 & 0.351 & 0.724 \\
P-value & 0.005 & 0.200 & 0.501 & 0.0310 & 0.001 & 0.316 \\
\hline
\end{tabular}

CCT, central corneal thickness.

Table V. Correlation between central corneal thickness and medial corneal optical density $(0-2 \mathrm{~mm})$.

\begin{tabular}{lcccc}
\hline Item (years) & Center & CCT & $r$ & P-value \\
\hline$<5$ & $16.20 \pm 2.56$ & $557.21 \pm 3.51$ & 0.137 & 0.462 \\
$5-10$ & $18.97 \pm 4.83$ & $569.41 \pm 4.01$ & 0.149 & 0.049 \\
$>10$ & $22.36 \pm 6.05$ & $581.72 \pm 3.17$ & 0.075 & 0.009 \\
\hline
\end{tabular}

CCT, central corneal thickness.

Table VI. Correlation between central corneal thickness and intimal corneal optical density $(0-2 \mathrm{~mm})$.

\begin{tabular}{lcccc}
\hline Item (years) & Posterior & CCT & $\mathrm{r}$ & P-value \\
\hline$<5$ & $20.37 \pm 7.61$ & $557.21 \pm 3.51$ & 0.221 & 0.036 \\
$5-10$ & $34.29 \pm 9.84$ & $569.41 \pm 4.01$ & 0.042 & 0.017 \\
$>10$ & $41.02 \pm 11.36$ & $581.72 \pm 3.17$ & 0.049 & 0.001 \\
\hline
\end{tabular}

CCT, central corneal thickness.

studies are available on diabetic keratopathy (7-11). Those few studies are carried out using confocal microscopy in the study of diabetic corneal neuropathy (7-11). Major clinical symptoms of diabetic retinopathy (DK) include corneal epithelium damages, decreased corneal perception, thickened corneal edema and nubecula, descemet's membrane folds, and corneal autofluorescence leakage and dry eye (3,7,12-14). Thus, hyperglycemia causes damage of different degrees to each layer of the corneal structure. Corneal optical density has a sensitive reflection on various traumas, inflammation and nubecula incurred by denaturation, and is widely used to describe corneal transparency. In the present study, we used the Pentacam to measure and identify changes in corneal optical density on each layer of the diabetics' cornea to reveal the pathogenesis of diabetic keratopathy.

Corneal optical density value in the diabetic and normal control groups. In the present study, we set the center point of the cornea as the object of reference and measured the optical density of each layer in each corneal section whose diameter was $<2,2-6,6-10$ and $10-12 \mathrm{~mm}$, including the superficial, middle, interior layers, and in total. We found that in the same section, the optical density increased from the centre to the periphery while in the same layer, the optical density decreased from the superficial layer to the interior layer. Such alterations were associated with the distribution of the corneal collagen fiber. Compared with the normal control group, the diabetics' corneal optical density in the same layer of the same section increased, and such an increase was most obvious in the middle and interior layer.

The finding indicated that the decline of transparency of the cornea was potentially due to the degeneration of the corneal collagen fiber leading to an increase in the corneal optical density. It has been previously shown that as patient age, the corneal collagen fiber was glycosylated and became thickened and hardened (15). The change in corneal collagen fiber and the extracellular matrix increased the corneal optical density. In a high-glucose environment, advanced glycation end products (AGEs) accumulated in the corneal epithelial basement membrane, or matrix. The descemet's membrane, which leads to abnormal protein cross-linking and damage of the cell structure, resulting in the clinical symptoms, includes local incrassation, multiple stratification, and discontinuity $(16,17)$. Thus, long-term hyperglycemia may lead to the degeneration of corneal collagen, leading to an increase in the corneal optical density and a decrease in transparency. In addition, damage of the pumping function of the corneal endothelium and the liquid epithelial barrier resulted in edema and nubecula, decreased transparency and increased optical density on the corneal stroma and epithelium. Corneal endothelial morphology becomes abnormal in patients with a long course of diabetes and their activity of $\mathrm{Na}^{+} / \mathrm{K}^{+}$-ATPase enzyme declines, resulting in pumping dysfunctions of corneal endothelium, edema and nubecula of corneal stroma and the increase of corneal optical density.

The normal cornea is a transparent tissue without blood vessels but full of nerve endings $(18,19)$. The central region of the cornea is associated with eyesight and has a relatively stable structure. Previous studies have shown that the thickness of the central regions is not associated with age (20). Thus, this region became the focus of the present study and the correlation of its optical density and the duration of diabetes was investigated. The results showed that as the duration of diabetes extended, the optical density in the middle and interior layer gradually increased. Therefore, the intimal and medial corneal optical density were positively correlated with the course of diabetes, while the optical density in the surface and total layer had no significant correlation with the course of diabetes. Thus, the transparency of the middle and interior layers of the cornea were the first to have abnormal changes, which indicated that 
in the early period of diabetes, the corneal endothelium and matrix may be the first to have lesions. It may be because of the dysfunction of corneal endothelial and the weakening of the water aspirator function (7,21-23). Thus, a correlation analysis was carried out between the course of diabetes and central corneal density and corneal endothelial cell count.

Correlation between central corneal thickness, corneal endothelial cell number, and corneal optical density. Through correlation analysis of the corneal thickness, corneal endothelial cell density and the course of diabetes, we found that as the duration of diabetes extended, the central corneal thickness increased while the corneal endothelial cell density decreased. However, the corneal endothelial cell density had no correlation with the stage of the disease. It was positively correlated with the central corneal thickness. Therefore, we conducted a correlation analysis between the central and intimal corneal optical density and central corneal thickness. The result showed that in the early period of diabetes ( $<5$ years), the medial and intimal corneal optical density had no correlation with central corneal thickness. In patients with long-term diabetes ( $>5$ years), the medial corneal optical density was positively correlated with central corneal thickness, while the intimal corneal optical density was positively associated with central corneal thickness in patients with short-term diabetes. Thus, in the early period of diabetic keratopathy, corneal optical density and central corneal thickness increased.

Generally speaking, the change of corneal thickness is an important indicator for a low corneal endothelial function. Apparent corneal incrassation indicated the malfunction of the cornea (20). The maintenance of corneal thickness depended on the degree of dehydration of strong water-absorbing base. It was mainly dependent on the epithelial liquid barrier and endothelial pump mechanism to maintain the cornea, and on the pumping function of the $\mathrm{Na}^{+} / \mathrm{K}^{+}$-ATPase enzyme of the endothelial cells to keep the balance between substrate ion and hydrodynamic force, in order that the cornea always be kept dehydrated and transparent $(7,11)$. When the activity of the $\mathrm{Na}^{+} / \mathrm{K}^{+}$-ATPase enzyme was reduced, the ionic pump function of the corneal endothelial cells was impaired and this was an important mechanism for diabetic corneal edema (24-27). Subsequently, corneal thickness was positively correlated with corneal intimal optical density. As corneal edema became thickened, corneal transparency was reduced and corneal optical density was increased accordingly (28). In terms of the course of the disease, intimal and medial corneal optical density was the most altered. Thus, intimal optical density was the first to become abnormal on diabetic keratopathy patients and this was associated with corneal endothelial dysfunction. The result of the present study showed that corneal endothelial cell density had no significant correlation with the stage of the disease. In the early period of diabetic keratopathy, the endothelial lesion was manifested in the abnormity of the morphology and function, and therefore a primary research orientation in the future.

In conclusion, in the early stage of diabetic keratopathy, corneal optical density in the interior and middle layers changed abnormally, and the change was closely associated with the duration of diabetes. The corneal thickness increased and central corneal thickness was positively correlated with the length of diabetes. The intimal corneal optical density was positively correlated with corneal thickness. Thus, the corneal optical density (especially the intimal optical density) is a sensitive indicator for diabetic keratopathy. Corneal optical density and central corneal density may be an important basis for the diagnosis of diabetic keratopathy.

\section{References}

1. Wang Y, Zhou Q and Xie L: Diabetic keratopathy: new progresses and challenges. Zhonghua Yan Ke Za Zhi 50: 69-72, 2014 (In Chinese).

2. Kaji Y: Prevention of diabetic keratopathy. Br J Ophthalmol 89: 254-255, 2005.

3. Cennamo G, Forte R, Aufiero B, La Rana A: Computerized Scheimpflug densitometry as a measure of corneal optical density after excimer laser refractive surgery in myopic eyes. J Cataract Refract Surg 37: 1502-1506, 2011.

4. Rosales P and Marcos S: Pentacam Scheimpflug quantitative imaging of the crystalline lens and intraocular lens. J Refract Surg 25: 421-428, 2009.

5. Lanza M, Cennamo M, Iaccarino S, Romano V, Bifani M, Irregolare $\mathrm{C}$ and Lanza A: Evaluation of corneal deformation analyzed with a Scheimpflug based device. Cont Lens Anterior Eye 38: 89-93, 2015.

6. Kolb $\mathrm{H}$ and Mandrup-Poulsen T: The global diabetesepidemic as a consequence of lifestyle-induced low-grade inflammation. Diabetologia 53: 10-20, 2010.

7. Takacs AI, Mihaltz K and Nagy ZZ: Corneal density with the Pentacam after photorefractive keratectomy. J Refract Surg 27: 269-277, 2011.

8. Hillenaar T, van Cleynenbreugel H, Verjans GM, Wubbels RJ and Remeijer L: Monitoring the inflammatory process in herpetic stromal keratitis: the role of in vivo confocal microscopy. Ophthalmology 119: 1102-1110, 2012.

9. Hamrah P, Cruzat A, Dastjerdi MH, Zheng L, Shahatit BM, Bayhan HA, Dana R and Pavan-Langston D: Corneal sensation and subbasal nerve alterations in patients with herpes simplex keratitis: an in vivo confocal microscopy study. Ophthalmology 117: 1930-1936, 2010.

10. Vivino MA, Chintalagiri S, Trus B and Datiles M: Development of a Scheimpflug slit lamp camera system for quantitative densitometric analysis. Eye (Lond) 7: 791-798, 1993.

11. Greenstein SA, Fry KL, Bhatt J and Hersh PS: Natural history of corneal haze after collagen crosslinking for keratoconus and corneal ectasia: Scheimpflug and biomicroscopic analysis. J Cataract Refract Surg 36: 2105-2114, 2010.

12. Fares U, Otri AM, Al-Aqaba MA, Faraj L and Dua HS: Wavefront-optimized excimer laser in situ keratomileusis for myopia and myopic astigmatism: refractive outcomes and corneal densitometry. J Cataract Refract Surg 38: 2131-2138, 2012.

13. Otri AM, Fares U, Al-Aqaba MA and Dua HS: Corneal densitometry as an indicator of corneal health. Ophthalmology 119: 501-508, 2012.

14. Constantinou M, Jhanji V, Tao LW and Vajpayee RB: Clinical review of corneal ulcers resulting in evisceration and enucleation in elderly population. Graefes Arch Clin Exp Ophthalmol 247: 1389-1393, 2009.

15. Kaji Y, Usui T, Oshika T, Matsubara M, Yamashita H, Araie M, Murata T, Ishibashi T, Nagai R, Horiuchi S and Amano S: Advanced glycation end products in diabetic corneas. Invest Ophthalmol Vis Sci 41: 362-368, 2000.

16. Konstantopoulos A, Kuo J, Anderson D and Hossain P: Assessment of the use of anterior segment optical coherence tomography in microbial keratitis. Am J Ophthalmol 146: 534-542, 2008 .

17. Konstantopoulos A, Hossain P and Anderson DF: Recent advances in ophthalmic anterior segment imaging: a new era for ophthalmic diagnosis? Br J Ophthalmol 91: 551-557, 2007.

18. Daxer A, Misof K, Grabner B, Ettl A and Fratzl P: Collagen fibrils in the human corneal stroma: structure and aging. Invest Ophthalmol Vis Sci 39: 644-648, 1998.

19. Wegener A and Laser-Junga H: Photography of the anterior eye segment according to Scheimpflug's principle: options and limitations - a review. Clin Experiment Ophthalmol 37: 144-154, 2009. 
20. Alsbirk PH: Corneal thickness. I. Age variation, sex difference and oculometric correlations. Acta Ophthalmol (Copenh) 56: 95-104, 1978.

21. Keoleian GM, Pach JM, Hodge DO, Trocme SD and Bourne WM: Structural and functional studies of the corneal endothelium in diabetes mellitus. Am J Ophthalmol 113: 64-70, 1992.

22. Cho YK, Chang HS, La TY, Ji D, Kim H, Choi JA and Kim MS Anterior segment parameters using Pentacam and prediction of corneal endothelial cell loss after cataract surgery. Korean J Ophthalmol 24: 284-290, 2010.

23. Buehl W, Stojanac D, Sacu S, Drexler W and Findl O: Comparison of three methods of measuring corneal thickness and anterior chamber depth. Am J Ophthalmol 141: 7-12, 2006.

24. Matsuda J, Hieda O and Kinoshita S: Quantification of corneal opacity after refractive corneal surgery using the anterior segment analyzer. Nippon Ganka Gakkai Zasshi 111: 447-453, 2007 (In Japanese).
25. Shankar H, Taranath D, Santhirathelagan CT and Pesudovs K: Anterior segment biometry with the Pentacam: comprehensive assessment of repeatability of automated measurements. J Cataract Refract Surg 34: 103-113, 2008.

26. Vemuganti GK, Reddy K, Iftekhar G, Garg P and Sharma S: Keratocyte loss in corneal infection through apoptosis: a histologic study of 59 cases. BMC Ophthalmol 4: 16, 2004.

27. Vesaluoma M, Pérez-Santonja J, Petroll WM, Linna T, Alió J and Tervo T: Corneal stromal changes induced by myopic LASIK. Invest Ophthalmol Vis Sci 41: 369-376, 2000.

28. Erie JC, Patel SV, McLaren JW, Hodge DO and Bourne WM: Keratocyte density in the human cornea after photorefractive keratectomy. Arch Ophthalmol 121: 770-776, 2003. 